\title{
Palliative care in nursing homes: a comparison of high- and low-level providers
}

\author{
Nancy A Hodgson, Amanda J Lehning
}

\begin{abstract}
The purpose of this study was to explore staff perceptions and concerns about the use of palliative care services in the nursing home setting. Six administrators from nursing homes were purposively selected for key informant interviews. Four common themes emerged, including issues related to the culture of care, the model of care, the relationships with hospice partners, and the role of staff. Recognition of staff perceptions is an important first step in improving the utilization of palliative care services. Staff insight provided clarification related to impediments in promoting a culture of care that was person-centered and relationshipbased. We conclude by identifying the solutions for raising the level of dialogue to promote palliative care practice in the nursing home environment.
\end{abstract}

Nancy A Hodgson is Acting Director and Senior Research Scientist,

Polisher Research Institute, Abramson Center for Jewish Life, North Wales, Pa, USA; Amanda J Lehning is Doctoral Student, University of California, Berkeley, CA, USA

Correspondence to: Amanda J Lehning Email: ajlehning@berkeley. edu
$\mathbf{T}$ There has been a significant amount of criticism during the past several years regarding the care of the dying in residential aged care facilities and all the while, the number of individuals dying in nursing homes has been increasing Approximately 22\% of all US deaths occur in nursing homes (Mitchell et al, 2005). This estimate is projected to increase to $40 \%$ by 2040 (Brock and Foley, 1998). Moreover, 50\% of older adults in the US are transferred from nursing homes to hospitals before they die (Levy et al, 2004). Nursing home residents are also more likely to die in pain or distress and have poor access to hospices (Hall et al, 2002; Teno et al, 2004; Hanson et al, 2005). Given these facts, the nursing home industry would appear to be in a unique position to provide palliative care.

In 1999, there were 1.6 million residents living in 18000 nursing facilities in the USA (Jones, 2002). Almost two-thirds of these facilities are under private, forprofit ownership, and more than $80 \%$ are certified to provide care to individuals with Medicare or Medicaid health coverage (Jones, 2002). Unlike other countries, such as the UK, the US federal government has imposed a number of regulations on the nursing home industry (Patterson et al, 2007). The Omnibus Budget Reconciliation Act of 1987 (OBRA 87) instituted individualized care plans and quarterly interdisciplinary team meetings to review each resident's care, imposed strict guidelines on the use of physical or chemical restraints (Lacey, 1999), created the Minimum Data Set (MDS), an assessment and information tracking form to determine whether nursing homes are in compliance with federal regulations (Streim et al, 2002). The OBRA 87 legislation also introduced staffing requirements, calling for all nurses' aides to be trained and certified, and required all nursing facilities with more than 120 beds to employ a full-time social services director (Lacey, 1999).

There is no mention within the OBRA 87 legislation, however, of palliative care programmes, and we estimate that less than $15 \%$ of Pennsylvania nursing homes engage in some type of palliative care programme (Hodgson et al, 2006). Most of the literature on end-of-life practice in US nursing homes features appalling statistics. For example, fewer than $10 \%$ of dying nursing home residents have had access to hospices (Miller et al, 2005). The reasons for such low utilization of palliative care is unclear, but it appears that nursing homes are frequently not aware of, or fail to consider, the added value of palliative care.

Previous research has primarily used survey methodology to generate lists of barriers. The prevailing barriers identified are the predominance of the curative model of medical care, lack of health care provider education and skill, and the inadequacy of reimbursement mechanisms (Miller et al, 2004; Rice et al, 
Box I. Criteria for determining level of palliative care services

I. Palliative care is included in the facility's annual strategic plan

2. Residents deemed eligible to receive palliative care are defined and communicated to those services

3. The presence of specific written policies and procedures in pain management

4. The presence of specific written policies and procedures related to palliative care standards in alternative/complementary therapies

5. Nursing home administrator reports that facility promotes and supports advance care planning

6. Facility has resident/family satisfaction form that includes questions regarding pain and symptom management

7. Facility acknowledges need for specialized palliative care services

8. Pain management offered as a palliative care staff development topic in the past year

9. Facility indicates pain management protocol American Geriatrics Society guidelines are present in facility training materials

2004; Stillman et al, 2005; Furman et al, 2007). The nursing home culture, with an emphasis on restorative care and increasing uses of technology, has found it difficult to strike a balance between working to provide a good death and complying with regulatory guidelines. Contrasting that stereotypical view, however, are a number of innovative case studies and grass root demonstration projects making their way into the literature (Katz, 2005; Kayser-Jones et al, 2005).

\section{Method}

The purpose of our investigation was to explore the challenges encountered in providing palliative care services to nursing home residents from the nursing home administrators' perspective. We relied on qualitative methodology because it was consistent with our study objectives. These were to identify the barriers perceived by nursing home administrators in providing palliative care services in Pennsylvania nursing homes, and to explore the meaning of the palliative care services for administrators that have been identified as either high-level providers or low-level providers of palliative care.

The project is the second phase of a two- part study. In part one, we surveyed nursing home administrators in Pennsylvania to describe existing services and classify them by level of care delivery (high, moderate or low) (Hodgson et al, 2006). The outcomes of interest in the larger study were specific characteristics of palliative care services and the level of service provision. Level of palliative care services was determined by the criteria outlined in Box 1.

In phase two, we purposively selected high and low providers from the larger sample to compare perceptions of barriers. Specifically, we adopted ethnography as our central methodological perspective (Hymes, 1974). The ethnographic interview consists of an in-depth, one-on-one conversation between an investigator and a respondent. It has long been a central data collection method in cultural anthropology (as well as other social and behavioral sciences) and the technical literature on it is extensive (Briggs, 1986; Gubrium and Holstein, 2002). Ethnographic interviews offer the opportunity for deeper, more individualized discussion that, in turn, permits the discovery and exploration of additional histories, perspectives and purposes that make up the experience of palliative care. The ethnographer was a socio-linguist whose 30 years of ethnographic research has focused on endof-life care practices in nursing homes. We explored administrators' experiences through their discourse.

The goal of the interviews was to obtain information from informed stakeholders about the barriers to providing palliative care services and initial thoughts on issues related to providing these services. Examples of questions were the following: 'From your perspective, what type of resident is an appropriate candidate for palliative care? Why? Give an example. What type of resident would be an inappropriate candidate, from your perspective? Why? Give an example.'

The interviews were completed with the nursing home administrators of six facilities, which were purposively selected because they were either highor low-level providers of palliative care services. The study was approved by the Institutional Review Board (IRB) of the Abramson Center for Jewish Life (formerly the Philadelphia Geriatric Center), the parent organization of the Polisher Research Institute. Each of the participating nursing homes consented to complete a survey documenting the palliative care services provided. Nursing homes were then clustered based on criteria previously described. Of the six high-level 


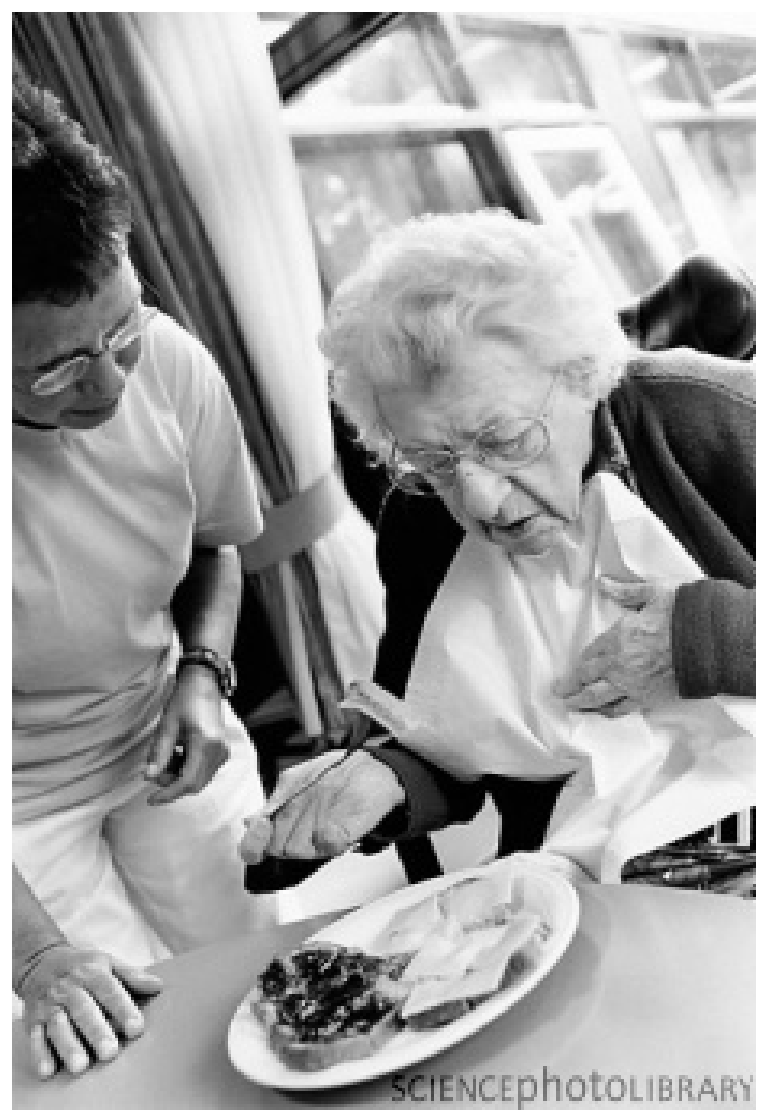

Person-centered care is an emerging and recurring theme in the gerontological literature

facilities contacted, three consented to participate. Of the ten low-level facilities contacted, three consented to participate. The six nursing homes represented a mix of urban, rural, small, large, profit and non-profit.

All interviews were audiotaped following procedures approved by the Abramson Center IRB. Subjects were assured that the information they provided would remain confidential and anonymous. Interviews were transcribed as soon as possible to ensure that the fine details of the interview and details of the recorded speech were documented. The transcribed text was analyzed using a variety of ongoing and overlapping qualitative analytic methods, including data inventory, coding, theme analysis, and semantic analysis. Because this project was discourse-based, close attention was paid to each participant's own words as a source of insight into behaviour and meaning. Socio-linguistic techniques were used to identify and explicate implicit or coded meanings in participants' statements (Werner and Schoepfle, 1987).

Analysis of the key informant interviews involved individual, in-depth reviews of recorded transcripts by the principal investigator and the ethnographer. Emerging themes from each nursing home were initially categorized based on the guided interview questions. The research team reviewed initial findings to assure consistency of thought and approach.

\section{Results}

Common themes were elicited across the six nursing homes and included issues related to the culture of care. The themes are discussed in opposites to highlight the differences between high-level facilities' and low-level facilities' orientation toward each dimension. The central barrier expressed was the traditional nursing home culture of care, with an emphasis on tasks and task completion, versus a person-centered care approach. This concept of culture of care was evident throughout the analysis of the discourse. Three sub-themes emerged within this overall theme. The first was the primary model of care identified by the administrator, whether medical or social. The second was the role of hospice and the formalization of partnerships with hospice providers. Lastly were issues around staffing with respect to skill level, training and perceived roles.

Person-centered care is an emerging and recurring theme in the gerontological literature. While there are many descriptions of person-centered practice, there are few models that define this concept. For the purpose of this study we used Kitwood's model $(1997,1998)$ to distinguish the culture of care. Kitwood developed his model from the idea that the biomedical perspective is inadequate when it comes to dementia care. $\mathrm{He}$ proposed a shift in the current culture from a medical model of care to a social model of care where the focus is not on illness and disease management, but rather on human relationships. In a social model the majority of staff time is spent interacting with residents, and teamwork defines the relationships among front-line staff.

\section{Culture of care discourse}

High-level nursing home administra- 
tors internalized and articulated the culture change values of relationship-based, person-centered care. The discourse and tone of voice of the participants conveyed that they believed in these concepts and wanted to incorporate them into care. High-level nursing home administrators also conveyed meaningful events through narrative rather than summarizing or generalizing. They spoke quite a bit about staff needs in the palliative care setting. The narrative of the discourse was more highly engaged, with a highly interactive orientation towards people that cut through the task orientation of lower level facilities.

'We've shifted our model due to a general acceptance of making the end of life as positive as possible as opposed to denying the inevitable death -and more acceptance of just letting people live the life they have- not going to the hospitals, having less done with diagnostic tests and invasive procedures.' (Administrator A: high level)

In contrast, in low-level homes the emphasis of the discourse was more characteristic of a traditional medical model of care with more emphasis on curative efforts. Hospice intervention was considered at the very end stage of life, and therefore not within the view of a palliative approach moving upstream. Here the roles of staff were more hierarchically structured For example, throughout the interviews with the low-level facilities, discourse was mechanistic and characterized by phrases such as 'the need to push staff' or 'I fight to prevent'. Nursing home administrators explained that the task-oriented culture is a barrier to staff participation but added that they could not find their way out.

'In nursing homes every regulation means that we have to maintain the person at their highest physical wellbeing.' (Administrator D: low level)

'We're so geared (and the regulations are, too) towards doing everything absolutely humanly possible to maximize our residents.' (Administrator E: low level)

\section{Model of care}

High-level facilities characterized the model of care as non-traditional, nonmedical, and more socially or relationship based. High-level nursing home administrators demonstrated a very personal relationship with direct care staff, describing a person-centered, relationship-based management style. These administrators' narratives and anecdotes revealed that they spent much of their time on the units, 'even when there weren't any problems or crises'. They knew the names of the staff, knew about their families and provided assistance to staff with their personal lives. Staff in these facilities recognized the individual needs of residents and the importance of providing a homelike environment. They viewed residents through a 'personal lens' and integrated the residents' preferences into their care.

'We do a very good job of addressing the medical things, but the three plights of residents are boredom, helplessness, and loneliness, and so we started our journey of changing our culture. We also put the decision-making in the hands of those working directly with residents, addressing emotional and social needs in addition to physical'. (Administrator B: high level)

In contrast, when discussing the model of care in the facility, the low-level facilities characterized their homes as medical or clinical. The curative focus of care was reflected in their reluctance to engage in discussions of end-of-life issues.

'We have a clinical model that's moving more towards a medical model.' (Administrator F: low level)

'The medical needs are quite great for the long-term care population. Our medical director is a certified medical director.' (Administrator E: low level)

\section{Hospice}

The high-level nursing homes described the hospice as a partner in care, and one small but important piece of the palliative care that they provide. They reported that they had worked hard to develop and maintain good relationships with their 
preferred hospice providers and took pride in what they described as the 'seamless transitions' between nursing home staff and hospice staff

'We asked the hospices for feed back. They told us that some facilities they go to staff are territorial and feel like hospice is intruding. But with our staff, they're partners, and they're all here for the same reason. So we include them in care plan meetings. They said they like coming here because we work with them as a team.' (Administrator B: high level)

When discussing the role of the hospice in their facility, the low-level homes often characterized palliative care as hospice alone. The hospice was described as taking over the care at end of life. This was also reflected in the nursing home staff at low-level facilities expressing divergent views from the hospices engaged at their facilities around goals of care.

'We start palliative care as soon as the staff say, "Hey, this person is terminal", then we let the family choose between one of three hospices. Of course, the length on hospice is always shorter than what hospices would like.' (Administrator D: low level)

'We primarily use the term hospice - I can't say we use the term end of life, we really don't use palliative care as a term. We call in hospice for 'palliative' treatment when someone is shutting down.' (Administrator F: low level)

\section{Role of staff}

The emphasis of the discourse on the role of staff members as described by the high-level homes was on the relationships that develop between the staff and the residents' and families. The level of staff participation in end-of-life care described was beyond that of traditional nursing staff. Roles were blurred and the traditional hierarchical structure was not in operation. There was an integration of staff and services. The administrators in high-level facilities were very focused on staff needs and had a more personal approach to care. Even in large facilities, the administrators knew the name of everyone in the building.

'Our mission is a mission of love. We make sure that we are hiring somebody who has love in the heart for the elderly.' (Administrator A: high level)

'The housekeeper, like, she wanted to do something very special for this woman, and she went out and got a flower. We have beautiful gardens here - and she brought in the flower and placed it over the top of the sheet covering the body. This resident was going to be transported to the morgue. And everybody just kind of stopped and looked...She said, "I wanted to honour her”. (Administrator C: high level)

In contrast, the discussion with the administrators in low-level facilities on the role of staff was characterized by an emphasis on reducing the high turnover and discipline specific roles. Consequently, low-level facilities related to resident and family end-of-life needs in terms of 'services' rather than of 'care', and were thus less mindful of the bigger picture than the high level facilities.

"We had someone appointed as a "hospice champion" in the building, our chaplain. He would bring up names during our clinical rounds.' (Administrator F: low level)

\section{Discussion}

To the extent that palliative care services can improve quality of care and quality of life for residents and families, it is important to explore the barriers perceived to providing these services. Our results uncovered several overarching themes, including the model of care, the role of hospice, and the role of staff. High-level facilities describe their model of care as non-medical and more relationship based. They also view the hospice as a partner in palliative care, and stress the importance of supportive relationships both among the staff and between staff and residents and families. In contrast, low-level facilities organize their facilities around the traditional medical model, equate palliative care with hospice care, and view staff in terms of their job-specific roles. 
The primary finding of interest was the parallel between the culture of the home and the level of palliative care. Broader acceptance of palliative care requires a shift in the culture of long-term care (Kristjanson et al, 2005). Advocates have identified a number of cultural barriers to providing adequate end-of-life care in nursing facilities, including inadequate support of staff caring for dying residents, the emphasis of regulations on restorative care (Ersek and Wilson, 2003; Froggatt et al, 2002) and a reluctance to collaborate with outside hospice providers (Zerzan et al, 2000). In addition, previous ethnographic and mixed-methods research has found that nursing facilities that incorporate the idea of caring for the dying individual into daily care processes and policies provide optimal palliative care (Travis et al, 2002; Forbes-Thompson and Gessert, 2005; Currow and Hogarty, 2006). In the current study, nursing home administrators in high-level facilities echoed these sentiments when they spoke of the need for the institution as a whole to value-person centered care.

One variable influencing the culture of care in aged residential settings was the level of education of the health care providers. Although nursing staff in long-term care are at the forefront of end-of-life services for individuals with dementia, they have not received the training to enable them to adopt palliative care practice. (Phillips et al, 2006; Phillips et al, 2007). Therefore, it is important to consider educational opportunities that are augmented by a supportive organizational culture to enhance palliative care practice.

The aim of the culture change movement is to change the organization of care provided in nursing homes so that it is more focused on the quality of relationships between the residents/families and staff at all levels (Ronch, 2003). Similar to the research on culture change movement we observed there was a lack of clarity about the most effective way to go about the process of implementing changes. In particular, there was a strikingly high reliance on trial and error and a strong need for role models in this area. Motivational problems cited included a lack of time, a lack of a champion, and a lack of external supports. Similar to earlier research on the discomfort nursing home staff may experience in terms of replacing restorative care with palliative care (Travis et al, 2002), several informants hinted that the motivational issues might be related to the perceived purpose: to not treat, to hasten death. The top-down approach is typical of this setting, but a bottomup approach would more likely result in success. For example, nursing home administrators aiming to implement organizational change may be more successful when they individualize resident care and solicit the participation of residents and families in organizational decisions (Scalzi et al, 2006). Empowering nurse aides to use their knowledge of individual residents and their families can lead to positive resident outcomes, particularly in terms of engagement in the life of the facility and personalized care (Applegate and Morse, 1994). Nurse aides are an invaluable source of information for administrators seeking to integrate individualized palliative care into the culture of the institution. Fostering a culture that embraces palliative care will remain difficult unless administrators reject a task oriented culture and learn to address the needs of their direct care staff.

\section{Limitations}

Given the small and select sampling of this study, further research will be needed to confirm the relative importance of these themes to other long-term care providers' perceptions of barriers to palliative services. Study findings, therefore, must be understood and interpreted within this context.

\section{Conclusion}

Access to palliative care is highly dependent on a complex interplay between individual, regional and macro-level forces (Blevins and Deason-Howell, 2002). Much of the earlier literature on barriers to palliative care has focused on the larger, macro-level concerns. Thus, further research will be needed to confirm the relative importance of the more micro-level themes identified in this study to the broader challenges in providing palliative care. The data from this study provided some evidence for the need to demystify this multidisciplinary approach to end of life care. If a palliative care philosophy is practiced throughout the long-term care industry, 
providers and family members might be more willing to use a hospice or palliative care programme.

Applegate M, Morse JM (1994). Personal privacy and interactional patterns in a nursing home. $J$ Aging Studies 8: 413-34

Blevins D, Deason-Howell LM (2002) End of life care in nursing homes: The interface of policy, research and practice. Behave Sci Law 20: 27186

Briggs C (1986) Learning How to Ask: A Sociolinguistic Appraisal of the

Role of the Interview in Social Science Research, Cambridge University Press, Cambridge

Brock DB, Foley DJ (1998) Demography and epidemiology of dying in the U.S. with emphasis on deaths of older persons. Hosp J 13: 49-60

Currow DC, Hegarty M (2006) Residential agedcare facility palliative care guidelines: improving care. Int J Palliat Nurs 12: 231-3

Dobbs DJ, Hanson L, Zimmerman S, Williams CS, Munn J (2006) Hospice attitudes among assisted living and nursing home administrators, and the long-term care hospice attitudes scale. J Palliat Med 9: $1388-400$

Ersek M, Wilson SA (2003) The challenges and opportunities in providing end-of-life care in nursing homes. J Palliat Med 6: 45-57

Forbes-Thompson S, Gessert CE (2005) End of life in nursing homes: Connections between structure, process, and outcomes. J Palliat Med 8: 545-55

Froggatt KA, Poole K, Hoult L (2002) The provision of palliative care in nursing homes and residential care homes: a survey of clinical nurse specialist work. Palliat Med 16: 481-7

Furman CD, Pirkle R, O’Brien JG, Miles T (2007)

Barriers to the implementation of palliative care in the nursing home. J Am Med Dir Assoc 8: $45-8$

Gubrium J, Hostein J (2002) Handbook of Interview Research: Context and Method. Sage, Thousand Oaks, CA

Hall P, Schroder C, Weaver L (2002) The last 48 hours of life in long-term care: a focused chart audit. I Am Geriatr Soc 50: 501-6

Hanson LC, Sengupta S, Slubicki M (2005) Access to nursing home hospice: perspectives of nursing home and hospice administrators. Palliat Med 8: 1207-13

Hodgson N, Landsberg L, Lehning A, Kleban M (2006) Palliative care services in Pennsylvania nursing homes. I Palliat Med 9: 1054-8

Hymes D (1974) Foundations in Sociolinguistics: An Ethnographic Approach. University of Pennsylvania Press, Philadelphia

Jones A (2002) The National Nursing Home Survey: 1999 summary. Vital Health Stat 13: $1-116$

Katz J (2005) Palliative care in residential care facilities: a brief review. Int J Palliat Nurs 11: 130-1

Kayser-Jones J, Chan J, Kris A (2005) A model long-term care hospice unit: care, community, and compassion. Geriatr Nurs 26: 16-20

Kitwood T (1997) The concept of personhood and its relevance for a new culture of dementia care. In: Miesen BML, Jones GMM (eds). Care-giving in dementia, research and applications volume 2. Routledge, London/New York: 3-13

Kitwood T (1998) Toward a theory of dementia care: ethics and interaction. J Clin Ethics 9: $23-$ 34

Kristjanson LJ, Walton J, Toye C (2005) End-oflife challenges in residential aged care facilities: a case for a palliative approach to care. Int I Palliat Nurs 11: 127-9

Lacey D (1999) The evolution of care: a 100-year history of institutionalization of people with Alzheimer's disease. J Gerontol Soc Work 31: 101-31

Levy CR, Fish R, Kramer AM (2004) Site of death in the hospital versus nursing home of Medicare skilled nursing facility residents admitted under Medicare's Part A Benefit. J Am Geriatr Soc 52:1247-54

Miller SC, Intrator O, Gozalo P, Roy J, Barber J, Mor V (2004) Government expenditures at the end of life for short- and long-stay nursing home residents: differences by hospice enrollment status. J Am Geriatr Soc 52:1284-92

Miller SC, Teno JM, Mor V (2004) Hospice and palliative care in nursing homes. Clin Geriatr Med 20:717-34

Mitchell SL, Teno JM, Miller SC, Mor V (2005) A national study of the location of death for older persons with dementia. J Am Geriatr Soc 53:299-305

Patterson SM, Hughes CM, Lapane KL (2007). Assessment of a United States pharmaceutical care model for nursing homes in the United Kingdom. Pharm World Sci 29: 517-25

Phillips JL, Davidson PM, Ollerton R, Jackson D, Kristjanson L (2007) A survey of commitment and compassion among nurses in residential aged care. Int J Palliat Nurs 13: 282-90

Phillips J, Davidson PM, Jackson D, Kristjanson L, Daly J, Curran J (2006) Residential aged care: the last frontier for palliative care. J Adv Nurs 55: 416-24

Rice KN, Coleman EA, Fish R, Levy C, Kutner JS (2004) Factors influencing models of end-oflife care in nursing homes: results of a survey of nursing home administrators. J Palliat Med 7: 668-75

Ronch J (2003) Changing institutional culture: Can we re-value the nursing home. In: Ronch JL, Goldfield JA (eds). Mental Wellness in Aging: Strengths-based Approaches. Health Professions Press, Baltimore, MD: 161-78

Scalzi CC, Evans LK, Hostvedt K (2006) Barriers and enablers to changing organizational culture in nursing homes. Nurs Adm Q 30: 368-72

Stillman D, Strumpf N, Capezuti E, Tuch H (2005) Staff perceptions concerning barriers and facilitators to end-of-life care in the nursing home. Geriatr Nurs 26: 259-64

Streim JE, Beckwith EW, Arapakos D, Banta P, Dunn R, Hoyer T (2002) Regulatory oversight, payment policy, and quality improvement in mental health care in nursing homes. Psychiatr Serv 53: 1414-96

Teno JM, Clarridge BR, Casey V, Welch LC, Wetle T, Shield R, Mor V (2004) Family perspectives on end-of-life-care at the last place of care. JAMA 29: 88-93

Teno JM, Kabumoto G, Wetle T, Roy J, Mor V (2004) Daily pain that was excruciating at some time in the previous week: prevalence, characteristics, and outcomes in nursing home residents. $J$ Am Geriatr Soc 52: 840-1

Travis SS, Bernard M, Dixon S, McAuley WJ, Loving G, McClanahan L (2002) Obstacles to palliation and end-of-life care in a long-term care facility. Gerontologist 42: 342-49

Watson J, Hockley J, Dewar B (2006) Barriers to implementing an integrated care pathway for the last days of life in nursing homes. Int J Palliat Nurs 12: 234-40

Werner O, Schoefle F (1987) Systematic Fieldwork: Foundations of Ethnography and Interviewing. Sage, Newbury Park, CA

Zerzan J, Stearns S, Hanson L (2000) Access to palliative care and hospice in nursing homes. JAMA 284: 2489-94 